\title{
The Physical Basis of Subtle Bodies and Near-Death Experiences
}

\author{
Deno Kazanis, Ph.D. \\ Center for Restorative Health and Improved Athletic \\ Ability Through Qi Movement, Lutz, FL
}

ABSTRACT: The discovery of "dark matter" may provide the key to an ageold mystery concerning the nature of humanity. "Dark matter" cannot be seen or felt, but composes at least 90 percent of the physical universe. Since it is not composed of charged particles, it cannot produce electromagnetic waves and can interpenetrate with our visible charge-carrying matter. I suggest that "dark matter" is the matter of mysticism, which constitutes the subtle bodies and was present before (and responsible for) the Big Bang that created visible matter.

"The more important fundamental laws and facts of physical science have all been discovered, and these are now so firmly established that the possibility of their ever being supplanted in consequence of new discoveries is exceedingly remote. . . . Our future discoveries must be looked for in the sixth place of decimals."

Albert Abraham Michelson, 1894

Mystical teachings throughout the ages and throughout the world provide a comprehensive explanation for phenomena such as neardeath experiences (NDEs). They describe persons as having a series of invisible interpenetrating bodies, often referred to as subtle bodies. Most, if not all, of the spiritual and philosophical writings and teachings that have emerged contain this concept. The ancient Egyptians, Chinese, and Greeks, as well as the Native Americans, the tribes of Africa, the Incas, early Christians, and Vedic seers of India all found the study of the visible and invisible bodies a key to the nature of humankind and the universe (Tansley, 1984). David Tansley wrote:

Deno Kazanis, $\mathrm{Ph} . \mathrm{D}$., is a biophysicist and Director of the Center for Restorative Health and Improved Athletic Ability through Qi Movement. Reprint requests should be addressed to Dr. Kazanis at 2310 Southern Lites, Lutz, FL 33549. 


\begin{abstract}
According to various esoteric schools, the Creation is divided into seven major planes of consciousness or matter. Man reflects this pattern through his manifestation upon the cosmic physical plane, and draws matter from the various levels in order to create bodies through which he can learn to express the Divinity which lies at the heart of his being. So common is this concept of gradations of consciousness and substance that references to it can be found in Christianity, Theosophy, Buddhism, Yoga, Judaism, Rosicrucianism, Sufism, as well as in the teachings of ancient Greece and Egypt. It is also to be found in the Zoroastrian religion and the spiritual beliefs of the Polynesians. An understanding of these inter-related planes of consciousness is basic to any study of the subtle anatomy of man. (1980, p. 80)
\end{abstract}

It is noteworthy not only that this belief in nonvisible "bodies" composed of matter from other "planes" has been described in considerable detail, but that these details are very consistent from culture to culture, from ancient to modern times. One must wonder about the coincidence and the persistence of such a conviction. But science has been unable to find any physical basis for such a belief; look as we will, no physical scientific evidence for these subtle bodies has been discovered. Are we to view subtle bodies as purely subjective concepts, perhaps useful in meditation, perhaps purely imaginary and dismissable as meaningless fantasies?

Mystics and shamans tell us that they have directly experienced these subtle bodies. Many traditions have speculated on the basis for these experiences, such as "energy fields" surrounding the body, but they fail to explain what could produce these fields physically. Nevertheless the belief in this phenomenon is very strong. Gopi Krishna spoke for many when he wrote:

It is high time now that scientists accept the existence of bioenergy (prana), the intelligent force behind all chemical actions and reactions of a biological organism. Here we deal with a new dimension of matter and consciousness. . . The experiments ... are yet in a rudimentary stage, but ... if the idea is based on a solid foundation, the experiments will be successful and the illusive medium will be located one day. (1975, p. 12)

Before I discuss a physical basis for this illusive medium and for NDEs, let me first briefly summarize a typical description of the subtle bodies as presented in Western mysticism. 


\section{The Subtle Bodies}

A person's bodies are usually described as interpenetrating "envelopes" or "sheaths" of decreasing densities (Mookerjee, 1991). The most dense is the "physical" body, which has two components, the very dense visible body and the less dense etheric body, which has a shape similar to the visible body. The etheric body is directly related to the nervous system, which it feeds, controls, and galvanizes into action. It appears as a fine network of energy webs or energy streams. This subtle body is a receiver, assimilator, and transmitter of "qi" or "prana," an internal energy (Tansley, 1984), and also contains the acupuncture meridians (Mookerjee, 1991).

The next interpenetrating body is referred to as the astral body and is composed of less dense matter from the astral plane. Involved with an interplay of emotions, it connects the mind by way of the etheric body to the external world. It can sense the mood of another person or "atmosphere" in a room or location. Next is the mental body, composed of even finer matter of the mental plane. It is used for rational, discriminative, and intellectual thinking (Tansley, 1984).

The soul, the vehicle of manifestation for the spirit, is veiled by these three bodies of mental, emotional, and dense "physical" matter. These three bodies are not regarded as permanent, but as lasting only one incarnation. The causal body, which contains the soul, is relatively permanent. Humankind is also composed of even finer matter from other planes (Tansley, 1984).

Most often there are seven primary "chakras," or energy centers, associated with the subtle bodies, and each chakra is associated with an endocrine gland and governs certain areas of the body. These chakras are (1) the base chakra, located at the base of the spine; (2) the sacral chakra, located below the naval; (3) the solar plexus chakra; (4) the heart chakra; (5) the throat chakra; (6) the "third eye" or brow chakra, located between the eyebrows, whose activation gives inner vision, a simultaneous knowledge of things as they really are, as the "third eye," cosmic consciousness, opens at this center; and (7) the crown chakra, located on the top of the head.

Through the chakras, the major nerve plexuses, the lesser ganglia and the intricate network of fine nerves, man registers those energies and forces which flow to him from a multitude of sources throughout the universe. (Tansley, 1984, p. 82) 
The subtle bodies are related to the gross or visible particles at several psychic points, which are interlinked by numerous subtle channels known as "nadis." The most important nadis are: (1) the central channel, which runs from just below the naval to the forehead; (2) a white channel, which runs on the left of the central channel; and (3) a red channel, which runs on the right of the central channel. The right and left channels run from the perineum at the base of the spine, spiraling in opposite directions around the central channel, and all three meet between the eyebrows (Mookerjee, 1991).

There is a special energy, or energies, associated with the subtle bodies, called "qi" in China, "prana" in India, and by other names in other cultures. In Chinese, qi

means air, power, motion, energy, life, etc. According to Chinese terminology the correct definition should be: "the intrinsic energy," or "the internal energy," or "the original energy, the eternal energy, the ultimate energy." (Liao, 1981, p. 26)

Qi can be cultivated through meditation or through specialized movements, such as tai chi chuan or qi gong. Cultivating qi brings health and general well being. An experiential phenomenon, qi or prana is an energy associated with the subtle bodies, which can also be utilized to arouse a potent inner energy referred to as "kundalini."

The existence of the subtle bodies would explain not only NDEs, but also out-of-body experiences (OBEs), clairvoyance and extrasensory perception, hands-on healing, the power of prayer, and comparable phenomena. But wherein lies the physical basis for these mystical bodies?

\section{“Dark Matter”}

Recent discoveries in physical cosmologists' explorations of the phenomenon of "dark matter" may well point the way to a physical basis for subtle bodies. Until recently scientists had no idea that this "dark matter" existed because it cannot be seen (thus its name) or touched, but can pass right through visible matter. These properties indicate that it is not composed of electrically charged particles. We know it exists only because of its gravitational effect observed in distant galaxies. Furthermore, "dark matter" is not rare; according to cosmologists, at least 90 percent of the universe is composed of it. The mystery of what this matter is and how to explore it still remains 
(Trefil, 1993). While I would prefer using some name other than "dark matter" for this phenomenon, the term has unfortunately crept into scientific literature and using any other term tends to confuse people. For this reason, I will continue to use the term in quotes.

The first scientific evidence for the existence of "dark matter" came when Fritz Zwicky (1933) showed that there wasn't nearly enough mass associated with visible light for gravity to hold the galaxies together in a region of space called the Virgo Cluster. This discovery was overlooked until the $1980 \mathrm{~s}$, when scientists, probing the universe with dedicated ground observatories and new instruments in space, discovered evidence of "dark matter" wherever they looked in the universe (Turner, 1993).

Exploration of this phenomenon has proceeded as a multidisciplinary effort involving physical cosmologists, particle physicists, astrophysicists, plasma physicists, and others, pursuing the detection of particle "dark matter" with the expectation that their search will reveal which model of particle physics is the most accurate: the Standard Model or some of its extensions, such as the Peccei-Quinn Symmetry, Supersymmetry, Technicolor, or Superstrings model. Each of these models proposes a different candidate for the identity of the "dark matter" particle, such as a very light axion, a light neutrino, and a heavy neutralino (Turner, 1993); however, it appears likely we need to introduce totally new candidates.

In addition, cosmologists and particle physicists hope that the determination of the "dark matter" particle will fill in some missing information on the Big Bang. The Big Bang is regarded as a single energy event whose eruption created not only matter but simultaneously time and space. Although the occurrence of the Big Bang is well established, science has failed to explain how an uncaused effect could have occurred; that is, science may be able to describe what happened but cannot explain why it happened.

I would like to make two points concerning "dark matter." First, the microwave background radiation of the universe (the "echo" of the Big Bang) and primordial nucleosynthesis (Big Bang atomic nuclear production) are the two quantitative tests supporting the hot Big Bang Theory (Schramm, 1993). Yet these two phenomena speak only to the creation of matter that produces electromagnetic radiation-luminous or visible matter-and not to "dark matter." Furthermore, since "dark matter" makes up most of the matter in the universe, it is possible that some forms of "dark matter" predate luminous matter, and could in fact be the substance out of which lu- 
minous matter was produced. That is to say, "dark matter" could be responsible for the Big Bang. The production of luminous matter in the Big Bang could have been generated from a very small percentage of the then-existing "dark matter." Thus, it would be possible to explore the nature of the universe prior to the Big Bang, and explore the properties and the role of "dark matter(s)" in the Big Bang.

Second, while we are exploring deep into the universe to comprehend "dark matter," there is also evidence here on earth for the presence of "dark matter." As mentioned, from ancient times to the present, humankind has experienced phenomena and developed concepts that suggest the presence of nonvisible "bodies" that are a part of us, often referred to as the subtle bodies (Tansley, 1984). These bodies could be composed of "dark matter," a substance capable of interpenetrating with our visible body, and are in every way a part of our being as much as our organs or our bones. There are considerable experimental data and experiential phenomena to support this view. These phenomena include the subtle bodies, qi or chi, out-ofbody experiences, NDEs, hands-on healing or therapeutic touch, and the power of prayer. Literature on these subjects abounds and so does scientific evidence; yet without a physical basis it has been largely ignored, if not ridiculed, by the scientific community. Now, however, the discovery of "dark matter" might well explain these phenomena. In order to understand how "dark matter" presents us with a physical basis for the subtle bodies, let us first consider a brief history of matter from Isaac Newton's time on.

\section{A Brief History of Matter}

The concepts of mass and matter appear to have been originally conceptually the same. Even when Newton introduced "mass" in his Laws of Physics he probably viewed mass and matter as one; and he probably thought that we saw and felt objects because they had mass. But Newton's Second Law of Motion, the inertial property of mass, gave it its first property or first definition. The equation $F=$ $\mathrm{ma}$, where $\mathrm{F}=$ force, $\mathrm{m}=$ mass, and $\mathrm{a}=$ acceleration, is the mathematical statement of Newton's Second Law. In this equation, the only known quantity is acceleration, which is defined as a change in velocity. What Newton did was to define "force" as that which causes acceleration, or changes an object's velocity (speed or direction). But he also defined "mass" as that which resists a change in motion, 
which gave rise to the inertial property of mass. It takes a greater force to produce an equivalent change of velocity of a more massive object.

Newton later gave mass another property when he "discovered" gravity. The universal attraction of the force of gravity is due to mass. This can be expressed in the equation $F=G M m / r^{2}$, where $F=$ the gravitational force, $M$ and $m=$ the two masses exerting the gravitational force on each other, $r=$ the distance between the two masses, and $G=$ some number or constant, which depends on the units of measurement used. This property of mass is very different from its inertial property. With gravity we are talking about an attractive force between two objects that have mass. As far as we know, these two properties are inseparable from mass, even though they are in principle conceptually different. In visible or luminous matter, the protons and neutrons in the atomic nucleus are primarily responsible for the gravitational force, because they have almost all of the mass of the atom.

In this century Albert Einstein was able to equate mass with energy. The equation $E=m c^{2}$, where $E=$ energy, $m=$ mass, and $c=$ the speed of light $\left(3 \times 10^{3}\right.$ meters/second). This expression not only showed that mass was a form of energy, but also stated that it takes a large amount of energy to produce a small piece of mass.

At the beginning, it was tacitly assumed that we saw mass and we touched mass. When we began exploring the quantity of electric "charge," and as electromagnetic theory was developed in the 19th century, our understanding of mass also changed significantly. Charge does not exert a force on mass, or vice versa. Charge exerts a force on charge, called an electromagnetic force; and mass exerts a force on mass, called a gravitational force. After the basic experimental equations for electromagnetic phenomena had been established, James Clerk Maxwell in 1873 discovered an inconsistency in these well-established equations unifying electric and magnetic phenomena; in correcting that inconsistency, the electromagnetic equations now known as Maxwell's Equations predicted that charge could generate electromagnetic waves that moved at the speed of light. Nothing like this had ever been suspected; Maxwell concluded that light was probably an electromagnetic wave.

As experimentation was performed to prove his theory, electromagnetic radiation was soon discovered everywhere, and was easily generated. Visible light was just a small part of the electromagnetic spectrum. Suddenly we discovered that light was a property of elec- 
tric charge; that is, we saw objects not because of their mass, but because of their charge. By the end of the 19th century most scientists believed that the last great discoveries in physics had been made, and only details needed to be worked on. However, the 20th century exploded with amazing new discoveries calling for unexpected new perspectives.

As the atom was explored and quantum mechanics was introduced, the importance of electric charge became even more profound. In fact, it turned out that the structure of the atom was electric in nature: negatively charged electrons attracted by a positively charged nucleus. Chemical phenomena were a property of charge, and most everyday phenomena became attributed to charge. Even the fact that we could hold an object in our hand was found to be a property of charge. Charge was responsible for vision and for the force that held electrons to the atom; it therefore gave the atoms their chemical properties, and so made them appear and feel solid. Of course, charged particles such as electrons and protons also had mass, but mass had nothing to do with seeing or feeling physical objects. It was the property of charge that made that possible.

If there were a massive object not made up of electrically charged particles, we could not see it, and it would pass right through visible matter. We wouldn't know it existed unless it were massive enough to exert a gravitational force on us. Mass clearly was not what we thought it was. It had become something different, having only the properties Newton mentioned, its inertial property and its gravitational property; and it was a form of energy, as Einstein showed. But mass itself could not be seen or touched.

With the discovery of "dark matter," which is not composed of electrically charged particles, we now have matter that cannot be seen or touched with our visible bodies. "Dark matter" and "luminous matter" can exist at the same place at the same time; they can interpenetrate. "Dark matter" could be an intricate part of us and we-the visible part of us- wouldn't know it.

\section{"Dark Matter" as the Matter of Mysticism}

Mysticism is its own discipline and is not dependent upon science for its verification. Those who have experienced phenomena associated with mysticism generally have no need for scientific proof. Nevertheless, because mass is a form of energy, it is reasonable that the 
subtle bodies would have a physical basis. The physical properties of "dark matter"-its invisible nature and its ability to interpenetrate with visible matter-are consistent with the physical properties of the subtle matter and the subtle bodies described by mystics, long before such a substance was even suspected by the scientific community. Let us take a look at phenomena such as NDEs and the power of prayer in light of "dark matter" subtle bodies.

Elisabeth Kübler-Ross (1975) and Raymond Moody (1975) independently found a convergence of experiential information from people who had NDEs. Such NDErs often claim that words are inadequate to explain their experience, but typically they report the same events. They say that they experienced leaving their "body" and observing themselves from a distance, witnessing in detail activities carried out; in some cases their observations could be confirmed. They say that their form may vary, from amorphous clouds to energy patterns or pure consciousness; others experience a distinct feeling of having a body, but one that is permeable, invisible, and inaudible to those in the phenomenal world. They report feelings ranging from fear and confusion to ecstatic feelings of timelessness, weightlessness, serenity, and tranquility. They say that they hear peculiar sounds, sometimes unpleasant, sometimes soothing. Many report passing through a dark enclosed space such as a tunnel, cave, funnel, or valley. They describe encounters with other beings such as dead relatives or friends, "guardian spirits" or "spirit guides"; visions of a light being who shows qualities of love, warmth, compassion, and a sense of humor are common. They often report communication through thought. They describe a life review, selfjudgment, or divine judgment; and an understanding of the consequences of their past actions and thoughts (Grof and Grof, 1980; Moody, 1975).

NDEs parallel the afterdeath views of esoteric traditions, including such different sources as Ruldolf Steiner (1968), Emanuel Swedenborg, and Tibetan Buddhism (Moody, 1975). NDEs and out-of-body experiences are easily explained by mystical subtle body models as the subtle body leaving the dense visible body for a period of time. The conditions that allow the separation to occur and the level at which this separation occurs-the etheric, astral, mental, or higher level-are not clear. In different cases the subtle body that separates may be different, and the experience may be accordingly unique and categorizable. The complete experience of the subtle bodies seems to be inaccessible to ordinary consciousness. 
Scientific evidence for the healing power of prayer and its effectiveness in medicine has recently been presented (Dossey, 1993). Statistically significant data and case histories present strong evidence for the power of prayer. However, regarding attempts to develop a physical model for prayer, Larry Dossey wrote:

Conventional forms of energy are an insufficient explanation for what we observe in spiritual healing experiments. In them the "energy" does not fade away with increasing distance, and it cannot be shielded, as we would expect if ordinary forms of energy were involved. (Dossey, 1993, p. 198)

Scientific evidence shows that praying can heal, "but we simply don't know how the mind of one person can engage in 'action at a distance' to bring about the healthful changes" (Dossey, 1993, p. 198). Dossey clearly pointed out the need to find and describe other physical systems that could effectively transport healing information.

I suggest that "dark matter" could present a model whereby information (energy) could be transported over distances unshielded by visible matter. The properties of "dark matter" in the form of the subtle bodies may allow directed information (energy) transport from one person's subtle body to another's. The role of the etheric body is to "act as a receiver, assimilator, and transmitter of prana" (Tansley, 1984, p. 23). The prana in turn may affect the gray matter of the brain or organs and stimulate action on the psychoneuroendocrine pathway or the psychoneuroimmunological pathway, or possibly other more direct pathways through the subtle body. "Hands-on" healing or therapeutic touch can operate similarly, by directly transferring information from one physical subtle body to another. Qi (chi) would be "dark matter" and/or the energy transmitted by "dark matter." The subtle bodies may supply a simple and direct vehicle for healing.

\section{Physical Properties of the Subtle Bodies}

With at least 90 percent of the matter in this universe such a great mystery, scientists today cannot state what is not possible. Depending on the properties of "dark matter(s)," things believed to be impossible today may become very ordinary tomorrow. From the phenomena described above, we can conclude a few things about "dark matter" and "dark matter" bodies. 
First, the physical properties of "dark matter" must be quite different from the physical properties of visible or luminous matter. The "sense" of time, space, and matter may be quite different, but related, requiring new concepts. Scientists think that the detection of "dark matter" will provide evidence for a "new physics," but they may not realize how "new" this physics may be. Second, "dark matter" must be composed of stable "atoms" or "particles" capable of manifesting into a structure, such as a subtle body. Third, "dark matter" can transmit and receive energy, allowing the transmission of some type of energy (qi) in a way analogous to but quite different from visible matter's transmission of electromagnetic waves.

Fourth, the "dark matter" of the type composing the subtle bodies would be nonbaryonic and without electrically charged particles, or it would surely have been noticed a long time ago, because in NDEs the body weight would then significantly diminish as the bodies separated. Mystics have always taught that the matter from the other planes was much less dense than the visible matter. The reason visible matter is so heavy is not due to charge or to electrons, but to nucleons-protons and neutrons-that make up the nuclei of atoms. These particles are unusually massive and are responsible for more than 99.95 percent of our mass. The electron is only $1 / 1,836$ as massive as the neutron or proton. It would appear that the "dark matter" of our bodies does not have these massive particles like nucleons, but is composed of much less dense particles that make up probably less than 0.1 percent of the mass in our body. But small masses can have very significant effects, as demonstrated by the low mass electron, which is responsible for nearly all electrical phenomena we observe.

Fifth, the subtle bodies must be composed of several unique forms of "dark matter," which make up the envelopes or sheaths of the subtle bodies of decreasing density. These subtle bodies are as much a basic part of our being as our organs or bones. Our total being would actually be a combination of interpenetrating "dark matters" and visible matter. The bodies somehow interact, forming a complete being.

Sixth, the "dark matter" bodies have their own senses-being able to hear and see during NDEs or out-of-body experiences-but it would appear that the these senses are much more expansive, capable of perception far beyond our normal experiences and therefore difficult to explain and verbalize. This may be due to a change in the sense of time, space and matter. 
Seventh, self-awareness moves with the "subtle bodies" during NDEs, suggesting that it has more to do with our self-awareness than does our visible body. Scientists have assumed and even attempted to prove that the mind is the source of self-awareness (Crick, 1994), but it is possible that the mind is more like a machine that can interact with the "dark matter" subtle bodies, and is not as such the creator of self-awareness. Paracelsus wrote:

Man has also an animal body and a sidereal body; and both are one, and are not separated. The relation between the two is as follows. The animal body, the body of flesh and blood, is in itself always dead. Only through the action of the sidereal body does the motion of life come into the other body. (Tansley, 1984, p. 23)

Eighth, the subtle "dark matter" bodies are capable of experiencing thoughts and emotions directly. Communication through thought is often mentioned in NDE accounts. With our dense physical bodies out of the way, the awareness of the activities of the mental and emotional bodies is more available. It appears that "dark matter," in the form of a subtle body, is capable of transmitting thought as an "energy" in a way perhaps similar to charge transmitting electromagnetic waves. With this transmission prayers and thoughts can be transmitted over distances, and benefit individuals through the subtle "dark matter" bodies, which in turn can affect the brain and organs of the visible body. C. W. Leadbeater wrote that thought has to be transferred through several sheaths or subtle bodies to reach the gray matter of the brain (Leadbeater, 1987), indicating the deeply unconscious nature of thought transfer. The "dark matter" bodies may be the physical manifestation of our subjective or unconscious "invisible" world.

Ninth, it would appear that qi or prana is primarily an energy property of the "dark matter" bodies, and not of luminous matter. Traditional sources classify qi into many categories, suggesting that $q i$ is a generalized term referring to different types of "dark matter" and/or the energy transmitted by "dark matter." Qi can be "cultivated" through meditation and movement, which can in turn affect the visible body. Healing, it would appear, can occur through actions performed on the visible body or actions performed directly on the subtle "dark matter" bodies. Many alternative approaches to health are techniques that affect the subtle bodies, which in turn affect the visible body. Acupuncture, although performed on the visible body, more directly affects the etheric subtle body, which in turn can affect 
the visible body. Qi movements operate similarly, affecting the subtle bodies directly, and then indirectly affecting the visible body.

Finally, we are not totally in touch with our subtle "dark matter" bodies in our "normal" state of mind. We may experience these subtle bodies more fully under unique conditions, or develop the ability through proper meditation and other techniques.

Insights into "dark matter" bodies may well be obtained through mystics, shamans, and esoteric teachers who have written books on this subject. Several books on kundalini describe the subtle bodies in considerable detail, informing the reader of this very significant phenomenon, both from an Eastern perspective (Mookerjee, 1991; Rinpoche, 1992) and from a Western view (Leadbeater, 1987; Steiner, 1989; Mindell, 1982; Tansley, 1984).

\section{Summary}

At the beginning of this paper I mentioned that mystical teachings from all cultures speak of the creation of the universe involving perhaps seven distinct types of matter and/or consciousness, and that humankind is composed of interpenetrating subtle bodies made up of these distinct types of matter. In the past there was no scientific basis for such an idea; yet today, with the discovery of "dark matter," we now know that there is matter in the universe that we cannot easily detect, and that can interpenetrate with our visible bodies. It is extraordinary that the mystical teachings have had such a concept as "interpenetrating matter," since until "dark matter" was discovered over the past 15 years, no such matter was thought to exist or was even suggested by anyone in the scientific community.

The universe may have been created by a sequence of events, terminating in the Big Bang of our visible matter, rather than a single energy event that created time, space, and matter. The transition from "no space," "no time," "no matter" to the Big Bang and our present awareness of time and space may have been a more gradual process involving several events, and each event may have involved a unique form of "dark matter" associated with a different sense of time and space. Art may be very important in the phenomenological exploration of this "dark matter," because it provides a multisymbolic language and serves as a bridge between the visible and the invisible, thus providing a portal of access. 
Scientists today believe that people are composed of matter created by the Big Bang and then processed into the atoms of the elements in the stars. This is true for our visible body, but in addition to this body, a much older tradition says that we also have subtle bodies composed of matters created at different stages in the development of the universe, which predate the Big Bang. If we are composed of "dark matter," if we have in addition to our visible body, subtle bodies or "sheaths" of interpenetrating unique forms of "dark matter," all of which in turn interpenetrate with our visible body, phenomena such as NDEs and out-of-body experiences can be regarded as physical experiences of our subtle "dark matter" bodies. From the point of view of physics, "dark matter" is just as real and just as physical as visible matter.

There is evidence dating back to the earliest recorded history of humans that there is more to the universe and there is more to us than we are aware of and can see with our ordinary eyes. Western science has pursued an exploration of the visible or luminous form of matter at the exclusion of other human experiences of more subtle matter. This focus has been double-edged, producing incredible scientific knowledge and technological achievements concerning visible matter, but unfortunately losing track of a much greater picture.

Now that "dark matter" has been discovered and our knowledge of electromagnetic radiation-producing matter is so great, we need to pursue an exploration of "dark matter" as vigorously as we have visible matter. We can no longer escape the conclusion that humankind is composed of more than we can see, and has several "dark matter" bodies that are a necessary part of us, but are not fully experienced in our daily life. Descriptions of this subtle body abound, and include literature from all ages and all cultures, too numerous to list, and scientific evidence for this phenomenon increases daily. The mystical teachings provide the clearest and most complete model for explaining both the experiential phenomena mentioned and the scientific evidence associated with them.

Physical scientists are certain that "dark matter" exists because they can observe the gravitational effects it produces. But they are uncertain about what this "dark matter" is, and have not yet considered the possibility of its existence before the Big Bang. Although the evidence for subtle "dark matter" bodies is substantial, physical scientists may still require hard physical evidence. We cannot easily detect "dark matter" with our luminous matter, with our instruments or machines, because the only physical property of "dark matter" the 
scientific community has recognized is its gravitational property. This might suggest that upon death a small but detectable weight loss might occur. But the etheric body, the astral body, and the mental body do not, as such, continue after death, and they are the most dense of the subtle bodies-though still not very dense relative to the visible body. These bodies may remain with the visible body and dissipate gradually, leaving the causal body, the soul, and still finer forms of matter to depart.

Weight losses on the order of a few ounces have been reported upon death, but the scientific value of these reports is questionable. Carlos Alvarado (1980) has reviewed efforts to detect subtle bodies and weight loss at death or during out-of-body experiences (OBEs). He concluded:

The evaluation of these old studies can be as difficult and ambiguous as that of more recent OBE experiments. Some of them can be criticized on methodological grounds. . . . In some cases it has not been possible to replicate the originally reported phenomena while in others there has been no attempted replication. Under these conditions the anomalous results of these studies, although sometimes impressive, do not have too much scientific value. Though the studies we have discussed fall short of presenting good instrumental evidence for the existence of an "astral body," they still may be of some interest. If nothing else, they provide a sense of historical continuity with modern studies and a reminder of the methodological difficulties of this type of work. (Alvarado, 1980, p. 7)

However, a recent news report in the London-based magazine I-to-I reported that German scientists had succeeded in weighing the human soul:

The human soul weighs $1 / 3,000$ th of an ounce, according to researchers at the Technical University of Berlin. They weighed over 200 terminally ill patients just before and immediately after they died. In each case, the weight loss was the same-1,3000th of an ounce.

In a letter in German science journal Horizon, Dr. Becker Mertens said his team had taken into account other possible explanations of weight loss (such as air leaving the lungs), but that "the inescapable conclusion is that we have now confirmed the existence of the human soul."

The challenge now was to "figure out exactly what the soul is composed of." Mertens believes the soul is a form of energy, but admits that attempts to identify this energy have been "unsuccessful to date." (Dallas, 1993, p. 11) 
Valuable clues for obtaining further physical evidence might be acquired through the writings on this subject from those who have had direct experience with the subtle "dark matter" bodies. Perhaps true mystics will in time be viewed as our first "dark matter" scientists, because they obtained objectivity with "dark matter" just as scientists have with visible matter. Currently, however, scientists know very little about the physical behavior of "dark matter," and because its sense of time and space may be different, it will not be easy to establish this physical evidence; yet as we move into the 21st century, it has the promise of an extraordinarily exciting adventure.

\section{References}

Alvarado, C. S. (1980). The physical detection of the astral body: An historical perspective. Theta, 8, 4-7.

Crick, F. (1994). The astonishing hypothesis. New York, NY: Macmillan.

Dallas, S. (1993, July-September). Human soul weighs $1 / 3,000$ th of an ounce. I-to-I, Issue 15, p. 11.

Dossey, L. (1993). Healing words. San Francisco, CA: HarperSanFrancisco.

Grof, S., and Grof, C. (1980). Beyond death. New York, NY: Thames and Hudson.

Krishna, G. (1975). The awakening of kundalini. New York, NY: Dutton.

Kübler-Ross, E. (1975). Foreword. In Moody, R. A., Life after life. Covington, GA: Mockingbird Books.

Leadbeater, C.W. (1987). Man visible and invisible. Wheaton, IL: Theosophical Publishing House.

Liao, W. (1981). Tai chi classics. Del Mar, CA: Golden Oak Promotions.

Mindell, A. (1982). Dreambody. Boston, MA: Sigo Press.

Moody, R. A. (1975). Life after life. Covington, GA: Mockingbird Books.

Mookerjee, A. (1991). Kundalini. Rochester, VT. Destiny Books.

Rimpoche, S. (1992). The Trbetan book of living and dying. San Francisco, CA: HarperSanFrancisco.

Schramm, D.N. (1993). Cosmological implications of light element abundances: Theory. Proceedings of the National Academy of Science, 60, 4782-4788.

Smoley, R. (1993). A gown both subtle and dense. Gnosis, No. 29, 10-13.

Steiner, R. (1968). Life between death and rebirth. Spring Valley, NY: Anthroposophic Press.

Steiner, R. (1989). An outline of occult science. Spring Valley, NY: Anthroposophic Press.

Tansley, D. V. (1984). The subtle body. New York, NY: Thames and Hudson.

Trefil, J. (1993, June). Dark matter. Smithsonian, pp. 27-35.

Turner, M.S. (1993). Dark matter: Theoretical perspectives. Proceedings of the National Academy of Science, 60, 4827-4834.

Zwicky, F. (1933). Die rotverschiebung von extragalaktischen nebein. Helvetica Physica Acta, 6, 110-127. 